\title{
Кондаков А.С. \\ Тепловая диагностика трения в шаровой опоре с полимерным антифрикционным вкладышем
}

Институт проблем нефти и газа СО РАН

(Россия, Якутск)

doi: $10.18411 / l j-30-11-2017-53$

idsp: 000001:lj-30-11-2017-53

\section{Аннотация}

Приводится расчётный метод восстановления функции интенсивности тепловыделения в шаровой опоре по температурным данным. Исследуется устойчивость метода к погрешностям в температурных измерениях. Приводится результат практической апробации метода для шаровой опоры автомобиля ВАЗ-2106 - «Жигули».

Ключевые слова: шаровая опора, мощность трения, интенсивности тепловыделения, температура, обратная задача, итерационная регуляризация, эксперимент.

\section{Abstract}

A calculation method for reconstructing the function of the intensity of heat release in a ball bearing from the temperature data is given. The stability of the method to the errors in temperature measurements is investigated. The result of the practical approbation of the method for the spherical support of the car VAZ-2106 - Zhiguli is given.

Keywords: spherical support, frictional power, heat dissipation, temperature, inverse problem, iterative regularization, experiment.

Предлагаемый метод тепловой диагностики узлов трения основан на следующем факте, что практически вся механическая энергия, затрачиваемая на трение, трансформируется в тепловую энергию $[1,2]$. Существующие методы непосредственного замера мощности трения предусматривают использование упругих элементов, установка которых, даже в условиях стендовых испытаний узлов трения, крайне затруднительно. Это приводит к необходимости определять механическую энергию, затраченную на трение, по замерам других величин. С точки зрения доступности измерений, наиболее выгодным является температурная информация, не требующая для измерения сложного и громоздкого оборудования.

Регистрация температуры в окрестности зоны трения, построение математической тепловой модели, адекватной процессу теплообмена в сопряжении, и решение соответствующей граничной обратной задачи позволяет восстановить теплоту, выделившуюся в результате трения $[3,4]$. Для решения трёхмерной граничной обратной задачи необходимо задавать замеры температуры на некоторой поверхности внутри одного из элементов сопряжения, что практически невыполнимо из-за возможного нарушения целостности материала и искажения температурного поля. Такое положение приводит к необходимости использования упрощённых математических тепловых моделей, которые достаточно адекватно описывают реальное температурное поле в сопряжении и значительно сокращают количество точек замера температуры, необходимых для восстановления функции тепловыделения при трении.

Чтоб показать принципиальную возможность проведения тепловой диагностики трения, рассмотрим одну из наиболее распространённых в технике узел трения шаровую опору с антифрикционным полимерным вкладышем. Шаровые опоры широко применяются в различных подвижных технических средствах в том числе автотранспортных. Для решения приведённой ниже обратной задачи теплообмена используется метод итерационной регуляризации на основе градиентных методов минимизации функционала [5]. 
Рассмотрим шаровую опору, расчётная схема которой приведена на рис.1. Металлический шаровой палец 1 совершает колебания с малой амплитудой и высокой частотой по поверхности вкладыша 2 из антифрикционного полимерного материала. Вкладыш жёстко соединён с металлическим корпусом 3.

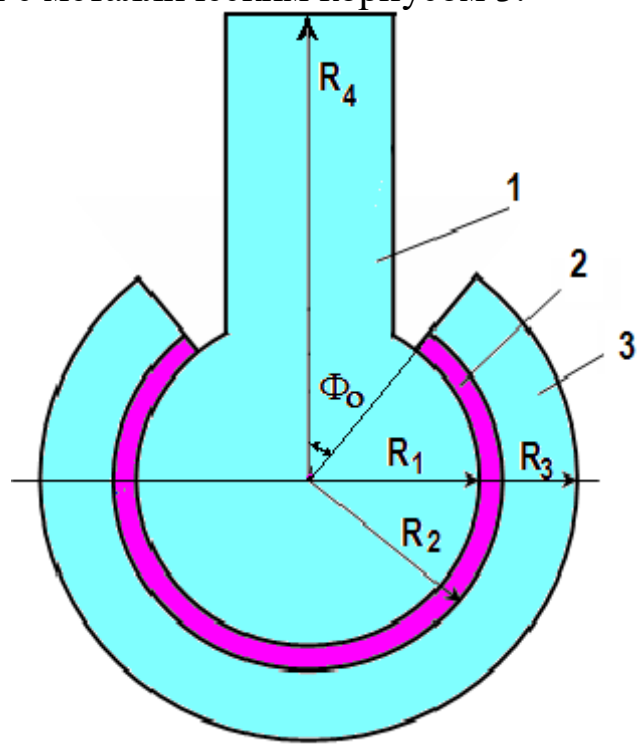

Рис.1. Схема шаровой опоры: 1 - палеи;;

2 - антифрикционный вкладыш; 3 - корпус

При допущении симметрии температурного поля шаровой опоры относительно оси нагружения, нестационарное температурное поле в шаровой опоре описывается двухмерным уравнением теплопроводности в сферической системе координат с сосредоточенным источником тепла на поверхности трения

$$
\begin{gathered}
C_{i}(T) \frac{\partial T}{\partial t}=\frac{1}{r^{2}} \frac{\partial}{\partial r}\left(\lambda_{i}(T) r^{2} \frac{\partial T}{\partial r}\right)+\frac{1}{r^{2} \sin \varphi} \frac{\partial}{\partial \varphi}\left(\lambda_{i}(T) \sin \varphi \frac{\partial T}{\partial \varphi}\right)+ \\
+\frac{\delta\left(r-R_{1}\right) \Theta(\varphi) Q(\varphi, t)}{4 \pi R_{1}^{2} \sin ^{2}(\varphi / 2)} ; \quad i=1,2,3 ; 0<t \leq t_{m} ; 0 \leq \varphi \leq \pi
\end{gathered}
$$

в котором индекс $i=1$ относится к материалу шарового пальца, $i=2$ - к полимерному композиционному материалу вкладыша, $i=3$ - к материалу корпуса. $Q(\varphi, t)$ - функция интенсивности тепловыделения в зоне трения шарового пальца и вкладыша; $\delta(x)=\left\{\begin{array}{ll}1, & x=0 ; \\ 0, & x \neq 0,\end{array} \quad \Theta(\varphi)= \begin{cases}0, & 0 \leq \varphi<\Phi_{o} ; \\ 1, & \Phi_{o} \leq \varphi \leq \pi .\end{cases}\right.$

Уравнение (1) дополняется начальным распределением температуры и граничным условием конвективного теплообмена на наружной границе расчётной области.

Обратная задача восстановления функции интенсивности тепловыделения $Q(\varphi, t)$ по температурным данным $f(\varphi, t)$, в точках расположенных во вкладыше по окружности при $r=R, R_{1}<R<R_{2} ; \Phi_{0} \leq \varphi \leq \pi$ заключается в минимизации функционала невязки

$$
J[Q(\varphi, t)]=\frac{1}{2} \int_{0}^{t_{m}} \int_{\Phi_{o}}^{\pi}[T(R, \varphi, t)-f(\varphi, \mathrm{t})]^{2} d \varphi d t
$$


на решениях уравнения (1) с заданными начальными и граничными условиями.

Решение поставленной обратной задачи проводится методом итерационной регуляризации на основе метода сопряжённых градиентов минимизации функционала невязки [5]. Градиент функционала (2) определяется решением сопряжённой краевой задачи.

Краевые задачи решались численно методом конечных разностей. В качестве объекта при выполнении расчётных вычислений была взята шаровая опора верхней передней подвески автомобиля ВАЗ-2106 - «Жигули» со следующими геометрическими размерами: $\mathrm{R} 1=18,5 \mathrm{mм}, \mathrm{R} 2=20 \mathrm{MM}, \mathrm{R} 3=25 \mathrm{мm}, \mathrm{R} 4=45 \mathrm{мм}, \Phi_{\mathrm{o}}=\pi / 4,5$.

C целью выявления практической пригодности метода тепловой диагностики трения в шаровой опоре был проведён вычислительный эксперимент по восстановлению заданной модельной функции $Q(\varphi, t)$, график которой приведён на рис.2(а). Решением прямой задачи были сохранены значения температур в точках вкладыша при $r=R, R_{1}<R<R_{2} ; \Phi_{0} \leq \varphi \leq \pi$, которые в последствии принимались как температурные данные для решения обратной задачи восстановления функции $Q(\varphi, t)$. Результат этого восстановления с точными температурными данными приведён на графике на рис.2(б). Видно, что точность восстановления достаточно высока за исключением окрестности конечной точки временного интервала, в которой градиент функционала невязки обнуляется и искомая функция не восстанавливается.

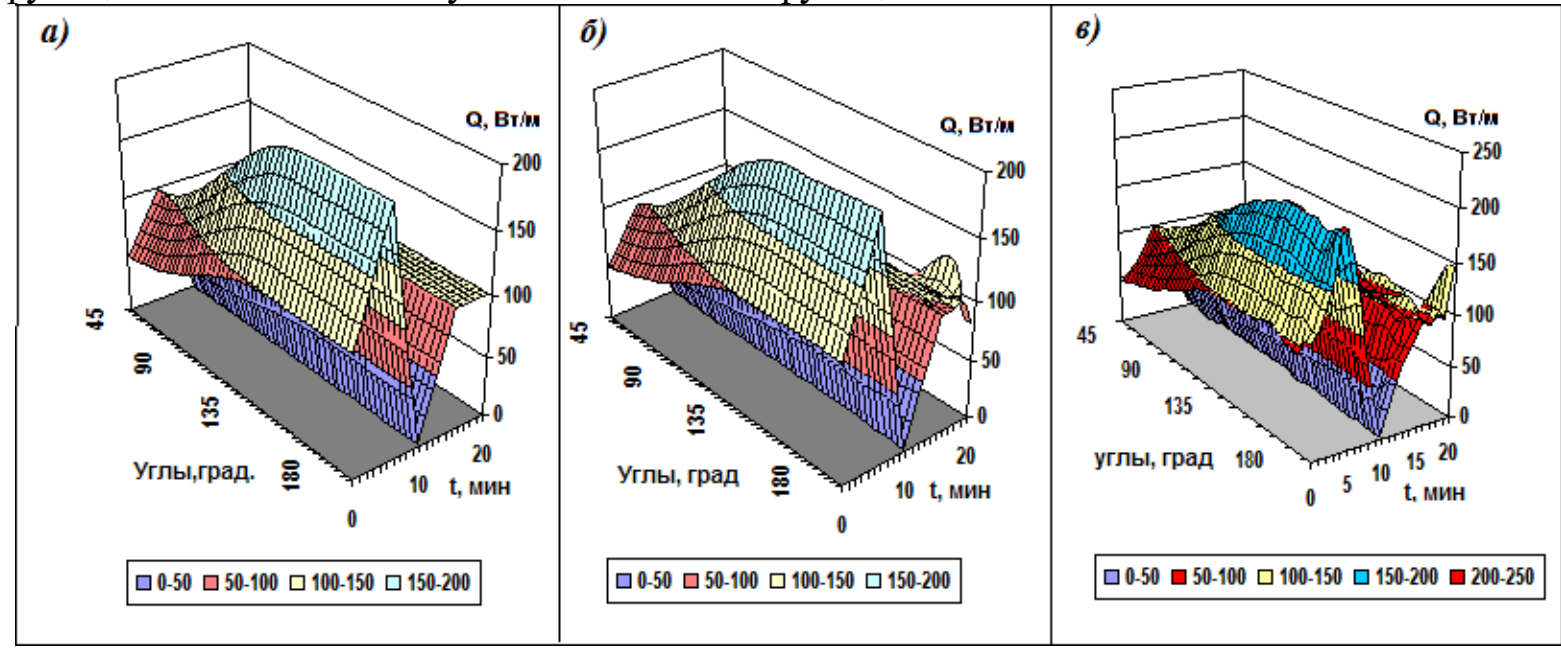

Рис.2. Восстановление функции интенсивности тепловыделения в шаровой опоре: а) - модельная функция;

б) - восстановленная функции по точным температурным данным; в) - восстановленная функция по возмущённым температурным данным.

Наибольший интерес представляет собой чувствительность предложенного метода восстановления интенсивности тепловыделения к погрешностям температурных данных, т.е. устойчивость алгоритма к возмущениям входных данных. Возмущения входных данных имитировались обычным образом

$$
\bar{f}(\varphi, t)=f(\varphi, t)+2 \Delta(\sigma(t)-0.5), \quad 0 \leq t \leq t_{m},
$$

где $\sigma(\mathrm{t})$ - нормально распределённая на отрезке $[0,1]$ случайная функция, $\Delta$ - уровень погрешности. На рис.2(в) приведён результаты расчёта восстановления при $\Delta=2$, т.е. вносится максимально 2 градуса Цельсия погрешности в температурных данных. Сравнения этих расчётных значений с точными модельными показывают достаточную устойчивость метода к погрешностям входных данных, приемлемую для практических целей.

Практическая апробация метода была проведена в эксперименте по восстановлению функции интенсивности тепловыделения в шаровой опоре верхней передней подвески автомобиля ВАЗ-2106 - «Жигули» во время её выезда по городу в 
ноябре месяце с температурой воздуха около -20 градусов Цельсия. Для этого заранее в антифрикционном полимерном вкладыше испытуемой шаровой опоры для регистрации температуры были закреплены шесть хромель-алюмелевых термопар равномерно по окружности на расстоянии 1 мм от зоны трения вкладыша с пальцем.

Для восстановления искомой функции интенсивности тепловыделения в шаровой опоре необходимо задавать температурные данные во всех точках дуги окружности внутри вкладыша, чего невозможно осуществить практически. В связи с этим использовали метод интерполирования температурных данных с установленных шести термопар. Результаты расчётов приведены на рис.3.

Во время контрольного заезда температурные датчики реагировали на изменение скорости автомобиля, на качество дорожного покрытия и на вынужденные остановки на светофорах. Там, где нагрузка на подвеску автомобиля возрастала, при движении по дорогам с неровным дорожным покрытием или при движении с высокой скоростью, регистрировалось повышение температуры в окрестности зоны трения в шаровой опоре. При движении по ровной дорожной поверхности или при остановках, например, на светофорах, регистрировалось понижение температуры в шаровой опоре. Все эти изменения в температурных данных реагировали соответственным образом на величину интенсивности тепловыделения.

Проведённые теоретические и экспериментальные исследования показывают практическую пригодность изложенной методики для определения затрат на трение в реальных узлах.

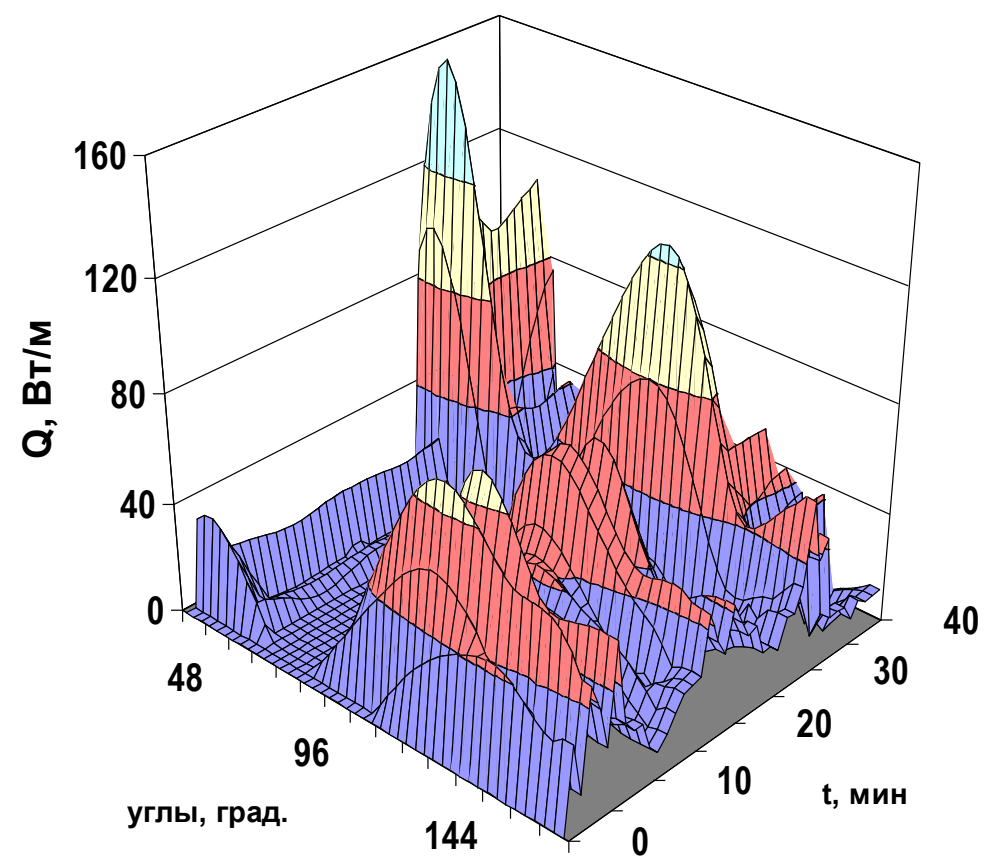

\section{$\square$ 0-40 $\square$ 40-80 $\square$ 80-120 $\square$ 120-160}

Рис.3. Восстановленная функиии интенсивности тепловыделения в шаровой опоре автомобиля $* * *$

1. Кузнецов В.Д. Физика резания и трения металлов и кристаллов: Избранные труды. - М.: Наука, 1977. $310 \mathrm{c}$.

2. Костецкий Б.И., Линник Ю.И. Энергетический баланс при внешнем тре-нии металлов // ДАН СССР. 1968. - Т.183, №5. - С. 42-46.

3. Bogatin O., Chersky I., Starostin N. Simulation and Identification of Nonstationary Heat Transfer in Nonuniform Friction Contact // Trans. ASME. Journal of Tribology. - 1993. - Vol. 115. №2. - P. 299-306.

4. Старостин Н.П., Тихонов А.Г., Моров В.А., Кондаков А.С. Расчет триботехнических параметров в опорах скольжения. - Якутск: Изд-во ЯНЦ СО РАН - 1999. - 274 с.

5. Алифанов О.М. Обратные задачи теплообмена. - Москва: Машиностроение. $-1988 .-280$ с. 